OPEN ACCESS

Edited by:

Neus Visa,

Stockholm University, Sweden

Reviewed by:

Tomas J. Ekstrom,

Karolinska Institutet, Sweden

Vladimir B. Teif,

University of Essex, United Kingdom

${ }^{*}$ Correspondence:

Kirsten C. Sadler

kirsten.edepli@nyu.edu

Specialty section:

This article was submitted to

Epigenomics and Epigenetics,

a section of the journal

Frontiers in Genetics

Received: 16 March 2017

Accepted: 22 May 2017

Published: 07 June 2017

Citation:

Madakashira BP and Sadler KC (2017) DNA Methylation, Nuclear

Organization, and Cancer.

Front. Genet. 8:76.

doi: $10.3389 /$ fgene.2017.00076

\section{DNA Methylation, Nuclear Organization, and Cancer}

\author{
Bhavani P. Madakashira and Kirsten C. Sadler* \\ Program in Biology, New York University Abu Dhabi, Abu Dhabi, United Arab Emirates
}

The dramatic re-organization of the cancer cell nucleus creates telltale morphological features critical for pathological staging of tumors. In addition, the changes to the mutational and epigenetic landscape in cancer cells alter the structure and stability of the genome and directly contribute to malignancy. DNA methylation is one of the best studied epigenetic changes in cancer, as nearly every type of cancer studied shows a loss of DNA methylation spread across most of the genome. This global hypomethylation is accompanied by hypermethylation at distinct loci, and much of the work on DNA methylation in cancer has focused on how local changes contribute to gene expression. However, the emerging picture is that the changes to DNA methylation in cancer cells has little direct effect on gene expression but instead impacts the organization of the genome in the nucleus. Several recent studies that take a broad view of the cancer epigenome find that the most profound changes to the cancer methylome are spread across large segments of the genome, and that the focal changes are reflective of a whole reorganization of epigenome. Hallmarks of nuclear reorganization in cancer are found in the long regions of chromatin marked by histone methylation (LOCKs) and nuclear lamina interactions (LADs). In this review, we focus on a novel perspective that DNA methylation changes in cancer impact the global structure of heterochromatin, LADs and LOCKs, and how these global changes, in turn, contribute to gene expression changes and genomic stability.

Keywords: chromatin, DNA methylation, lads, Large Organized Chromatin Lysine Modifications (LOCKS), cancer

\section{INTRODUCTION}

For over a century, pathologists have used cellular morphology to guide their diagnosis of cancer. Chief among the morphological features that distinguish benign from malignant cells is the shape, size, structure and composition of the nucleus (Zink et al., 2004). Moreover, nuclear morphologies are often pleiotropic across a single tumor, reflecting the heterogeneous nature of cancer. Altered nuclear morphology also reflects broad changes in genome positioning and epigenetic changes which occur during transformation. We review recent data showing that widespread epigenetic changes are among the most prominent and common features of the cancer nucleus.

Chromatin organization is dictated by interactions between DNA and nuclear structural proteins, such as nuclear lamins, and by epigenetic modifications. These modifications and changes to nuclear structure are key features distinguishing cancer cells from their normal counterparts. Moreover, changes to genome structure and epigenetic changes also can be tumorigenic, by causing genomic instability, a hallmark of cancer (Hanahan and Weinberg, 2011). 
Here, we discuss data showing that three epigenetic marks that occupy large regions of the genome are changed in cancer. First, cytosine methylation $(5 \mathrm{mC})$ of DNA is among the most well studied epigenetic modifications, global loss of DNA methylation is a common feature of cancer (Feinberg and Vogelstein, 1983a; Gama-Sosa et al., 1983). Recent work has shown that the pattern of DNA hypomethylation in cancer is characterized by long regions termed Partially Methylated Domains (PMDs) (Lister et al., 2009; Hon et al., 2012), accompanied by massive disruption in nuclear organization. The Lamina Associated Domains (LADs) (Guelen et al., 2008) and the long stretches of the genome termed Large Organized Chromatin lysine ("K") modification (LOCKs) (Wen et al., 2009; McDonald et al., 2011) differ markedly between cancer and normal cells (McDonald et al., 2011; Timp et al., 2014). Exciting work has uncovered significant overlap between PMDs, LADs and LOCKs (Timp and Feinberg, 2013; Timp et al., 2014), suggesting that regional, not local, changes are the defining features of the cancer epigenomic landscape.

\section{DNA METHYLATION: FEATURES, FUNCTIONS AND CHANGES IN CANCER}

DNA methylation was the first epigenetic change to be reported in cancer (Feinberg and Vogelstein, 1983a; Gama-Sosa et al., 1983; Ehrlich et al., 1985). Over 80\% of CpGs are methylated in human somatic cells, these are concentrated in repetitive sequences in intergenic regions and introns, whereas the $\mathrm{CpG}$ islands (CGI) found in the promoters of most genes (Lister et al., 2009; Roadmap Epigenomics et al., 2015) are protected from methylation (Deaton and Bird, 2011). This pattern is largely constant across somatic cell types from the same organism, with less than $20 \%$ of all CpGs showing any changes in methylation across cell types (Lister et al., 2009; Ziller et al., 2013; Zhang et al., 2016).

Pioneering work showing the essential role of DNA methylation in silencing imprinted genes (Reik et al., 1987; Sapienza et al., 1987; Swain et al., 1987; Li et al., 1993), inactivating X chromosome (Mohandas et al., 1981) and repressing repetitive DNA to prevent transposon activation (Chandler and Walbot, 1986; Schwartz and Dennis, 1986) coincided with the discovery of DNA hypomethylation in cancer (Feinberg and Vogelstein, 1983a; Gama-Sosa et al., 1983). These converged into a model proposing that DNA methylation regulated expression of oncogenes and tumor suppressors. Thousands of studies have pursued this theory; while some convincingly show a direct and inverse relationship between gene expression and DNA methylation of a regulatory region, most do not. In fact, most methylated CpGs reside in intergenic regions and most of the differentially methylated regions (DMRs) between normal and cancer cells are in these regions (Baylin and Jones, 2011). Moreover, the DMRs in cancer are largely not focal, but are instead spread across broad regions of the genome (Figure 1). In some cases, these DMRs represent a partial loss of methylation across a large region (i.e., PMD). Thus, the highly cited examples where methylation of a regulatory region controls gene expression in cancer appear to be the exception rather than the rule. We postulate that methylome reorganization in cancer reflects a massive change in the distribution of heterochromatin and nuclear organization, and that this reorganization can indirectly control gene expression.

\section{The Cancer Methylome and Nuclear Organization}

The methylome landscape in normal cells is that of broad peaks across long stretches of intergenic repeat-rich regions and valleys in CGIs and CpG poor regions (Figure 1). A birds-eye view of the methylome reveals that the large hypermethylated peaks, become hypomethylated in several cancer types (Varley et al., 2013; Ziller et al., 2013; Landau et al., 2014). Peaks of methylation in normal cells are characterized by stretches with over $80 \%$ methylation of most CpGs. These are converted to PMDs (Figure 1; Hansen et al., 2011), defined as a $5 \mathrm{~kb}$ to $10 \mathrm{Mb}$ region with average $50 \%$ CpG methylation (Lister et al., 2009). The emergence of PMDs essentially flattens the methylation landscape in cancer cells, blurring the boundaries between what in normal cells constitute methylation valleys (CGIs) and peaks (CGI shores). The novel concept here is that cancer DMRs (cDMRs) are largely comprised of PMDs, and that the focal regions of differential methylation need to be viewed in the context of the methylation level of the entire region of the genome. An additional novel idea from these studies is that the dramatic change in the cancer methylome is reflective of a randomization of methylation patterns, whereby each cytosine is methylated in some cells and not methylated in others (Hon et al., 2012; Landau et al., 2014; Timp et al., 2014).

The importance of PMDs in cancer was highlighted by a pioneering study focused on "CpG shores." These are $2 \mathrm{~kb}$ away from CGIs, cover genes expressed in a tissue-specific manner, and are variably methylated during tissue differentiation (Irizarry et al., 2009). While most of the methylome is static, shores are the most variable across cell types (Figure 1). Interestingly, cDMRs are also found largely in CpG shores (Hansen et al., 2011). Indeed, the location of cDMRs reflects the cell of cancer origin, possibly because these are the regions of the methylome that are most amenable to change.

\section{DNA Methylation and Cancer: Cause or Consequence?}

Genomic instability is carcinogenic. Since regions of DNA hypomethylation in cancer cells correspond with hotspots of chromosomal breaks (Eden et al., 2003; Rodriguez et al., 2006; Irizarry et al., 2009) and regions of the cancer genome where the methylation pattern appears random are more prone to mutation (Landau et al., 2014) suggests a link between DNA hypomethylation and genomic instability. Moreover, repressing transposons is a central function of DNA methylation, and transposon activation can contribute to genomic rearrangements by retrotransposition (Helman et al., 2014; Tubio et al., 2014). Contrasting data from the analysis of 51 premalignant lesions which showed that large blocks of hypomethylation that are found in advanced cancers are detected even in lesions that are not considered prone to malignant transformation 


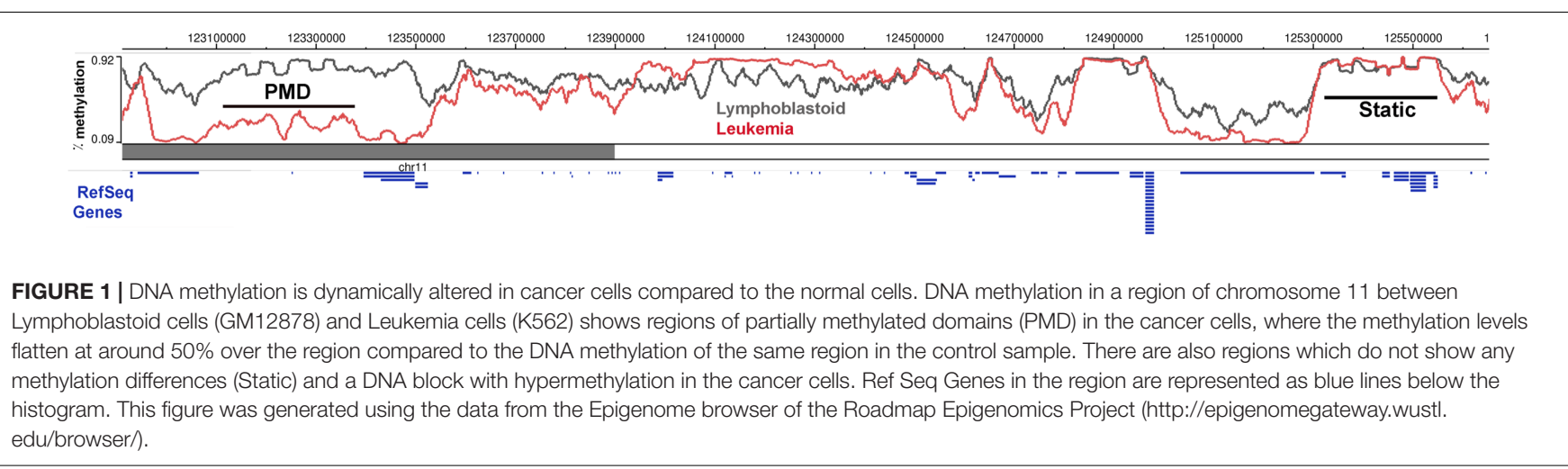

(Timp et al., 2014). Further study is required to determine whether DNA hypomethylation can cause cancer in all cell types.

Data from model organisms supports the hypothesis that loss of DNA methylation is oncogenic. In mice, a strong hypomorphic allele of the DNA methyltransferase, Dnmt1, causes DNA hypomethylation and genomic instability leading to aggressive T-cell lymphoma (Gaudet et al., 2003) and heterozygosity for this allele synergized with $N f 1$ and Tp53 to accelerate sarcoma formation (Eden et al., 2003). Similarly, a hypomorphic allele of Dnmt3b in combination with defects in a DNA repair gene caused lymphoma (Trinh et al., 2002). Our work in zebrafish (Mudbhary et al., 2014) demonstrated that overexpression of the epigenetic modifier, UHRF1, caused liver cancer in the absence of any other sensitizing mutations. In this model, UHRF1 overexpression caused global DNA hypomethylation and chromatin reorganization which first caused senescence as a tumor suppressive mechanism and when senescence was bypassed, tumors formed. We speculate that senescence induced by UHRF1 overexpression may be related to reorganization of the nuclear lamina, which causes senescence in other systems (Criscione et al., 2016).

\section{NUCLEAR ORGANIZATION AND CHROMATIN DOMAINS: LADS AND LOCKS}

Nuclear lamins have essential roles in maintaining nuclear structure, organizing chromosome territories (Cremer et al., 2006), interacting with nuclear actin (Burke and Stewart, 2013) and regulating gene expression (Reddy et al., 2008). A major role for lamins is to interact with distinct regions of the genome (i.e., LADs) covering $35-40 \%$ of the mammalian genome in blocks ranging from 0.1 to $10 \mathrm{MB}$ (Guelen et al., 2008; Meuleman et al., 2013; Amendola and van Steensel, 2015). LOCKs were first defined by long stretches of histone H3 lysine 9 dimethylation (H3K9me2) which largely overlap with LADs (Guelen et al., 2008; Hawkins et al., 2010; Peric-Hupkes et al., 2010; Hon et al., 2012; Luperchio et al., 2014; Figure 2). Thus, LOCKs represent blocks of the genome which are packaged into repressive chromatin structures. Interestingly, PMDs in cancer largely correspond to regions of LOCKs and LADs (Pujadas and Feinberg, 2012; Luperchio et al., 2014; Figure 2).

\section{LADs and TADs}

Most transcription occurs in the center of the nucleus; regions of the genome relegated to the nuclear periphery and encompassed in LADs are generally repressed (Dekker, 2008). Experimental repositioning of genes by targeting them to the nuclear envelope transmembrane proteins leads to repression of key genes involved in myogenesis, demonstrating the importance of gene positioning for tissue specific gene regulation (Robson et al., 2016). Similarly, depletion of B- type lamins in Drosophila de-repressed genes at the nuclear periphery (Shevelyov et al., 2009).

Lamina interactions are AT rich, containing epigenetic marks $\mathrm{H} 3 \mathrm{~K} 9 \mathrm{me} 2$, reduced $\mathrm{H} 3 \mathrm{~K} 36 \mathrm{me} 3$, with the LAD borders enriched for CTCF, H3K4me3 and H3K27me3 (Guelen et al., 2008; Zullo et al., 2012; Meuleman et al., 2013; Harr et al., 2015). Similar to the finding that most regions of DNA methylation are static across cell types, many LADs also tend to be constitutive across cell types (cLADs), although some are cell type specific. (Guelen et al., 2008; Meuleman et al., 2013). cLADs range from $100 \mathrm{~kb}$ to $10 \mathrm{Mb}$ and have low gene density (Meuleman et al., 2013), whereas variable LADs (vLADs) tend to contain developmentally regulated genes, and their position in the nucleus is altered by developmental cues (Zullo et al., 2012; Harr et al., 2015). Interestingly, many vLADs are enriched for GAGA motif containing sequences, called Lamina Associated Sequences (LASs), which are important for positioning regions of the genome at the nuclear periphery, as shown by experiments that artificially integrated LASs randomly throughout the genome (Zullo et al., 2012; Harr et al., 2015). LASs can be recognized by specific proteins that function to shape LAD architecture or recruit proteins such as the Polycomb Repressor Complex 2 (PRC2), which mediates the repressive H3K27me3 mark (Zullo et al., 2012; Harr et al., 2015). As expression of PRC proteins are deregulated in several cancer types (Luperchio et al., 2014), it is an intriguing possibility that LAD mediated targeting of PRC2 to tumor suppressors could cause their repression in cancer.

The borders of LADs are enriched in binding sites for the chromatin organizer CCCTC- binding factor (CTCF) which, along with SMC- family complex- Cohesin binds to the CTCF 

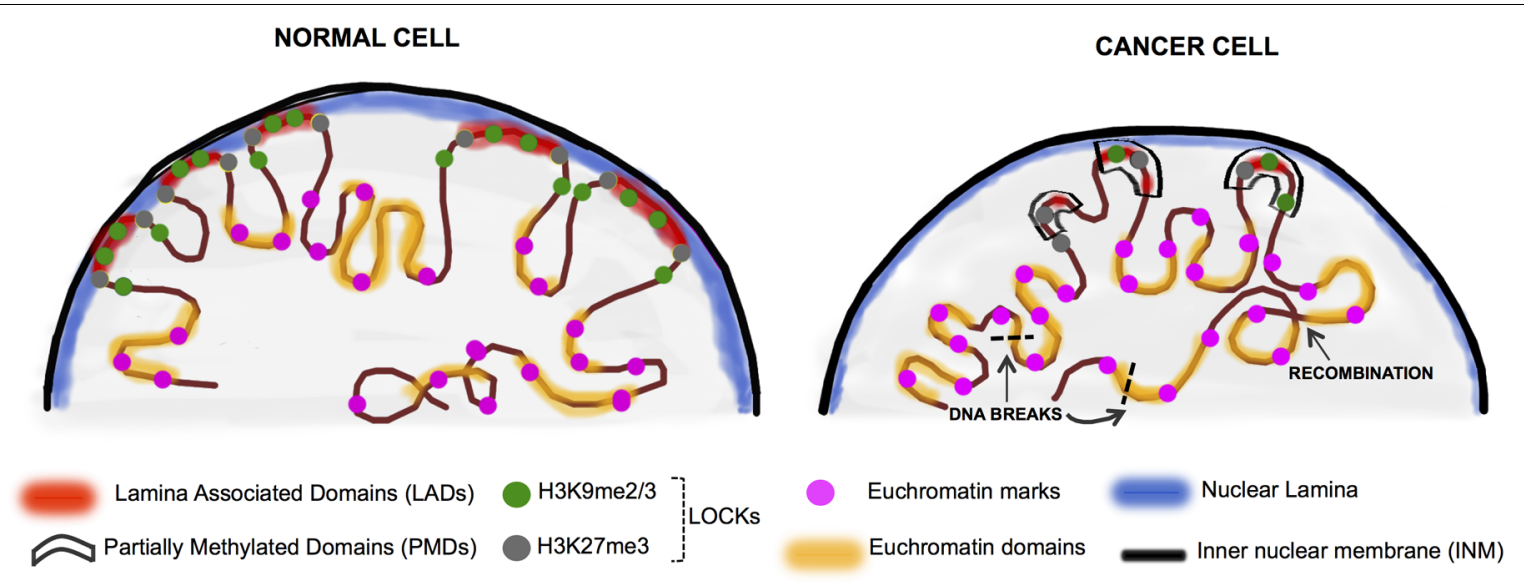

FIGURE 2 | Chromatin rearrangement in the cancer nuclei. In normal differentiated cells, heterochromatin is organized in the nuclear periphery by binding to the nuclear lamina proteins (in blue) and is organized into LADs (red) which overlap significantly with LOCKs (H3K9me2/3 and H3K27me3-green and gray circles). The active domains are tagged by the euchromatin histone marks (pink circles). Cancer cells display nuclear chromatin rearrangement with decreased lamin expression in the lamina, increased euchromatinization, and significant loss of LADs and LOCKs. In cancer, large blocks of DNA termed PMDs (black boxes) coincide to a large extent with LADs and LOCKs. These events can ultimately lead to random DNA recombination events as well as the less stable open chromatin are hotspots for DNA breaks (black dashed lines) (Kind et al., 2013; Luperchio et al., 2014; Feinberg et al., 2016).

recognition motif across the genome and acts as an insulator, thus regulating genomic stability, tissue- specific expression and overall epigenetic homeostasis (Ong and Corces, 2014). CTCF binding sites are found in the borders of both LADs and Topologically Associating Domains (TADs) (Luperchio et al., 2014). While LADs comprise heterochromatic regions, TADs can either be A-type (open, gene-rich chromatin) or B- type (closed, gene - poor) (Achinger-Kawecka and Clark, 2017). While TADs are fairly stable between different cell types, changes within TADs occur during development and differentiation (Luperchio et al., 2014). The relationship between LADs and TADs and the relevance to cancer is an area of active investigation.

\section{LOCKs}

Large Organized Chromatin Lysine Modifications were first described as large heterochromatic domains enriched for $\mathrm{H} 3 \mathrm{~K} 9 \mathrm{me} 2$ and associated with repressive heterochromatin (Wen et al., 2009). LOCKs comprise $>45 \%$ of the genome of liver cells, and $30 \%$ of differentiated ES cells, whereas less than $5 \%$ of genome is defined as a LOCK in undifferentiated ES cells (Wen et al., 2009). More recent studies also describe blocks of other repressive histones (H3K9me3 and H3K27me3), which occur at negligible levels in ES cells but expand during differentiation, supporting the general model of LOCKs/ blocks as highly dynamic heterochromatic domains during differentiation (Hawkins et al., 2010; Peric-Hupkes et al., 2010). Studies investigating the relationship between LOCKs and gene expression have shown that in liver cells, genes localized within LOCKs are generally silenced, while the same genes were found outside LOCKs in the brain and were expressed. Also, many genes that were not expressed were encompassed by LOCKs in both tissues, showing that LOCKs are strongly correlated with tissue-specific gene silencing (Wen et al., 2009; McDonald et al., 2011).
Interestingly, there is a high degree of overlap between LADs and LOCKs (Wen et al., 2009; McDonald et al., 2011). However, the functional relationship between these chromatin domains is unknown. Elegant studies show LAD sequences become localized to the nuclear rim even when integrated into a non-LAD locus, and this positioning within the nucleus is $\mathrm{H} 3 \mathrm{~K} 9 \mathrm{me} 2 / 3$ and H3K27me3 dependent (Zullo et al., 2012; Harr et al., 2015). Another study found that when the methyltransferase that deposits H3K9me2/3 (G9a) was suppressed in cancer cells, LADs "loosen," since they exhibit less heterochromatin at the nuclear rim (Zullo et al., 2012; Harr et al., 2015) suggesting that changing one of these domains may affect the other.

\section{LOCKS, LADS AND DNA METHYLATION TRANSFORM THE CANCER NUCLEUS}

Studies on defining the structural and functional relationships between epigenetic marks and nuclear organization are relatively recent, and thus there are few reports of how these work together in cancer. The frequent overlap of $\mathrm{H} 3 \mathrm{~K} 9 \mathrm{me} 3$ and $5 \mathrm{mC}$ (Rose and Klose, 2014) suggests that LADs have heterochromatin promoting epigenetic marks, such that both the nuclear position and the epigenetic decorations dictate expression, however, further studies are required to determine how universal this is. Some exciting new findings linking DNA methylation, histone methylation and the nuclear lamina suggest that LADs, LOCKs and PMDs overlap in cancer cells reflecting broad reorganization of the genome in the nucleus (Timp and Feinberg, 2013; Rose and Klose, 2014).

In many types of cancer, widespread rearrangement or loss of LOCKs and LADs has been reported (Wen et al., 2009; Berman et al., 2011; Hansen et al., 2011; McDonald et al., 2011). Additionally, the large blocks of hypomethylated DNA found in 
cancer cells correspond to LADs and LOCKs (Berman et al., 2011; Hansen et al., 2011, 2014; Timp and Feinberg, 2013; Timp et al., 2014). A study comparing colorectal adenocarcinomas to normal tissues from the same patients supports this model: $30 \%$ of the genome had profound DNA hypomethylation in large blocks corresponding to LADs and LOCKs (Hansen et al., 2011). This changed the sharply delimited methylation boundaries at $\mathrm{CpG}$ islands and shores, creating novel hypomethylation domains in CG-dense regions (Berman et al., 2011). In particular, the PMDs that characterize the repatterning of the cancer methylome are integrated with the changes in nuclear organization (Feinberg et al., 2016). One study found euchromatin islands within LAD/LOCK regions enriched for DNase hypersensitive sites and differentially methylated (Wen et al., 2012) suggesting that the very nature of LOCKs and LADs as primarily repressive domains is redefined in cancer. Since these domains are defined by the method used to isolate them: LADs by association with the nuclear lamina (Guelen et al., 2008), and LOCKs by a long stretch of H3K9Me2 (Wen et al., 2009), and these marks often overlap (Luperchio et al., 2014), it is possible that these are not entirely two different domains of chromatin, but instead reflect approaches that identify the same genomic architectural feature. This suggests that the differences in LADs and LOCKs between cancer and normal cells reflect an extensive reorganization of nuclear and genome structure.

How do changes to chromatin structure impact the cancer cell phenotype? One possibility is a direct impact on gene expression. Recently, it was discovered that PMDs in cancer contain nearly one third of the Transcription Start Sites (TSS) and correspond to LADs and LOCKs (Berman et al., 2011). Interestingly, nearly all the regions of CGIs hypermethylation are encased within large regions of hypomethylation, so that average levels of methylation are the same across the region (Figure 1). This essentially eliminates the rationale to focus on small regions of hypermethylation as candidate gene regulatory domains. Indeed, one exciting study has shown that genes with promoters which have the most random (or disordered) methylation pattern are generally not expressed or show wide divergence in expression across samples (Landau et al., 2014). Instead, these large PMDs or regions of disordered methylation that overlap with LOCKs and LADs likely change the 3D organization of chromatin, moving genes within them to the nuclear periphery and silencing them These wide scale changes can result in entire regions of the genome moving from repressive domains to accessible domains, potentially changing their transcriptional status by the fact of their position, but not directly because the methylation at specific $\mathrm{CpGs}$ in the genes are altered.

The finding that methylation changes appear even before cancer development and that the methylome is progressively

\section{REFERENCES}

Achinger-Kawecka, J., and Clark, S. J. (2017). Disruption of the 3D cancer genome blueprint. Epigenomics 9, 47-55. doi: 10.2217/epi-2016-0111

Amendola, M., and van Steensel, B. (2015). Nuclear lamins are not required for lamina-associated domain organization in mouse embryonic stem cells. EMBO Rep. 16, 610-617. doi: 10.15252/embr.201439789 changed as cancer progresses (Feinberg and Vogelstein, 1983a,b; Hansen et al., 2011) supports this as a potential cause of cancer. Additionally, largely euchromatinized DNA is more prone to breaks (Falk et al., 2008). Moreover, it is proposed that the reduction to 50\% methylation in PMDs suggests that methylation becomes disordered, with a stochastic methylation of every $\mathrm{CpG}$ in every cancer cell which is indicative of cancer heterogeneity.

\section{SUMMARY AND FUTURE DIRECTION}

While the changes in nuclear structure in cancer have been appreciated for decades, studies integrating nuclear organization and cancer epigenetics are relatively new and it remains unknown how the epigenetic landscape influences the three dimensional organization of the cancer nucleus. Although DNA hypomethylation and massive changes to histone marks and gene positioning characterizes most cancer cells, how these changes occur is not known. New perspectives on the genome organization in cancer requires adjustment of the model where epigenetic changes at discrete loci are interrogated to find their direct role as a regulator of cancer gene expression. Changes in chromatin structure may contribute to the genomic instability that is a hallmark of most cancers, yet the mechanism of this important change is not yet clear. Finally, how other aspects of the epigenome, including the distribution of histone variants and other epigenetic features, interact with the LADs, LOCKs and PMDs remains an important focus for future study.

\section{AUTHOR CONTRIBUTIONS}

$\mathrm{BM}$ and KS together researched and authored this mini review titled "DNA Methylation, Nuclear Organization and Cancer" for the Epigenomics and Epigenetics special topic "Design and principles of nuclear structure."

\section{FUNDING}

This work is supported by grant 5R01DK080789 from the NIH.

\section{ACKNOWLEDGMENT}

We are indebted to members of the Sadler lab for helpful discussions and in particular to Elena Magnani for critical reading of the manuscript.

Baylin, S. B., and Jones, P. A. (2011). A decade of exploring the cancer epigenome biological and translational implications. Nat. Rev. Cancer 11, 726-734. doi: $10.1038 / \mathrm{nrc} 3130$

Berman, B. P., Weisenberger, D. J., Aman, J. F., Hinoue, T., Ramjan, Z., Liu, Y., et al. (2011). Regions of focal DNA hypermethylation and long-range hypomethylation in colorectal cancer coincide with nuclear lamina-associated domains. Nat. Genet. 44, 40-46. doi: 10.1038/ng.969 
Burke, B., and Stewart, C. L. (2013). The nuclear lamins: flexibility in function. Nat. Rev. Mol. Cell Biol. 14, 13-24. doi: 10.1038/nrm3488

Chandler, V. L., and Walbot, V. (1986). DNA modification of a maize transposable element correlates with loss of activity. Proc. Natl. Acad. Sci. U.S.A. 83, 1767-1771.

Cremer, T., Cremer, M., Dietzel, S., Muller, S., Solovei, I., and Fakan, S. (2006). Chromosome territories-a functional nuclear landscape. Curr. Opin. Cell Biol. 18, 307-316. doi: 10.1016/j.ceb.2006.04.007

Criscione, S. W., De Cecco, M., Siranosian, B., Zhang, Y., Kreiling, J. A., Sedivy, J. M., et al. (2016). Reorganization of chromosome architecture in replicative cellular senescence. Sci. Adv. 2:e1500882. doi: 10.1126/sciadv.1500882

Deaton, A. M., and Bird, A. (2011). CpG islands and the regulation of transcription. Genes Dev. 25, 1010-1022. doi: 10.1101/gad.2037511

Dekker, J. (2008). Gene regulation in the third dimension. Science 319, 1793-1794. doi: $10.1126 /$ science. 1152850

Eden, A., Gaudet, F., Waghmare, A., and Jaenisch, R. (2003). Chromosomal instability and tumors promoted by DNA hypomethylation. Science 300:455. doi: $10.1126 /$ science. 1083557

Ehrlich, M., Zhang, X. Y., and Wang, R. Y. (1985). Human DNA methylation: methylated DNA-binding protein, differentiation and cancer. Prog. Clin. Biol. Res. 198, 255-269.

Falk, M., Lukasova, E., and Kozubek, S. (2008). Chromatin structure influences the sensitivity of DNA to gamma-radiation. Biochim. Biophys. Acta 1783, 2398-2414. doi: 10.1016/j.bbamcr.2008.07.010

Feinberg, A. P., Koldobskiy, M. A., and Gondor, A. (2016). Epigenetic modulators, modifiers and mediators in cancer aetiology and progression. Nat. Rev. Genet. 17, 284-299. doi: 10.1038/nrg.2016.13

Feinberg, A. P., and Vogelstein, B. (1983a). Hypomethylation distinguishes genes of some human cancers from their normal counterparts. Nature 301, 89-92.

Feinberg, A. P., and Vogelstein, B. (1983b). Hypomethylation of ras oncogenes in primary human cancers. Biochem. Biophys. Res. Commun. 111, 47-54.

Gama-Sosa, M. A., Slagel, V. A., Trewyn, R. W., Oxenhandler, R., Kuo, K. C., Gehrke, C. W., et al. (1983). The 5-methylcytosine content of DNA from human tumors. Nucleic Acids Res. 11, 6883-6894.

Gaudet, F., Hodgson, J. G., Eden, A., Jackson-Grusby, L., Dausman, J., Gray, J. W., et al. (2003). Induction of tumors in mice by genomic hypomethylation. Science 300, 489-492. doi: 10.1126/science. 1083558

Guelen, L., Pagie, L., Brasset, E., Meuleman, W., Faza, M. B., Talhout, W., et al. (2008). Domain organization of human chromosomes revealed by mapping of nuclear lamina interactions. Nature 453, 948-951. doi: 10.1038/nature06947

Hanahan, D., and Weinberg, R. A. (2011). Hallmarks of cancer: the next generation. Cell 144, 646-674. doi: 10.1016/j.cell.2011.02.013

Hansen, K. D., Sabunciyan, S., Langmead, B., Nagy, N., Curley, R., Klein, G., et al. (2014). Large-scale hypomethylated blocks associated with Epstein-Barr virus-induced B-cell immortalization. Genome Res. 24, 177-184. doi: 10.1101/ gr.157743.113

Hansen, K. D., Timp, W., Bravo, H. C., Sabunciyan, S., Langmead, B., McDonald, O. G., et al. (2011). Increased methylation variation in epigenetic domains across cancer types. Nat. Genet. 43, 768-775. doi: 10.1038/ng.865

Harr, J. C., Luperchio, T. R., Wong, X., Cohen, E., Wheelan, S. J., and Reddy, K. L. (2015). Directed targeting of chromatin to the nuclear lamina is mediated by chromatin state and A-type lamins. J. Cell Biol. 208, 33-52. doi: 10.1083/jcb. 201405110

Hawkins, R. D., Hon, G. C., Lee, L. K., Ngo, Q., Lister, R., Pelizzola, M., et al. (2010). Distinct epigenomic landscapes of pluripotent and lineage-committed human cells. Cell Stem Cell 6, 479-491. doi: 10.1016/j.stem.2010.03.018

Helman, E., Lawrence, M. S., Stewart, C., Sougnez, C., Getz, G., and Meyerson, M. (2014). Somatic retrotransposition in human cancer revealed by wholegenome and exome sequencing. Genome Res. 24, 1053-1063. doi: 10.1101/gr. 163659.113

Hon, G. C., Hawkins, R. D., Caballero, O. L., Lo, C., Lister, R., Pelizzola, M., et al. (2012). Global DNA hypomethylation coupled to repressive chromatin domain formation and gene silencing in breast cancer. Genome Res. 22, 246-258. doi: $10.1101 /$ gr.125872.111

Irizarry, R. A., Ladd-Acosta, C., Wen, B., Wu, Z., Montano, C., Onyango, P., et al. (2009). The human colon cancer methylome shows similar hypo- and hypermethylation at conserved tissue-specific $\mathrm{CpG}$ island shores. Nat. Genet. 41, 178-186. doi: 10.1038/ng.298
Kind, J., Pagie, L., Ortabozkoyun, H., Boyle, S., de Vries, S. S., Janssen, H., et al. (2013). Single-cell dynamics of genome-nuclear lamina interactions. Cell 153, 178-192. doi: 10.1016/j.cell.2013.02.028

Landau, D. A., Clement, K., Ziller, M. J., Boyle, P., Fan, J., Gu, H., et al. (2014). Locally disordered methylation forms the basis of intratumor methylome variation in chronic lymphocytic leukemia. Cancer Cell 26, 813-825. doi: 10.1016/j.ccell.2014.10.012

Li, E., Beard, C., and Jaenisch, R. (1993). Role for DNA methylation in genomic imprinting. Nature 366, 362-365. doi: 10.1038/366362a0

Lister, R., Pelizzola, M., Dowen, R. H., Hawkins, R. D., Hon, G., Tonti-Filippini, J., et al. (2009). Human DNA methylomes at base resolution show widespread epigenomic differences. Nature 462, 315-322. doi: 10.1038/nature08514

Luperchio, T. R., Wong, X., and Reddy, K. L. (2014). Genome regulation at the peripheral zone: lamina associated domains in development and disease. Curr. Opin. Genet. Dev. 25, 50-61. doi: 10.1016/j.gde.2013.11.021

McDonald, O. G., Wu, H., Timp, W., Doi, A., and Feinberg, A. P. (2011). Genomescale epigenetic reprogramming during epithelial-to-mesenchymal transition. Nat. Struct. Mol. Biol. 18, 867-874. doi: 10.1038/nsmb.2084

Meuleman, W., Peric-Hupkes, D., Kind, J., Beaudry, J. B., Pagie, L., Kellis, M., et al. (2013). Constitutive nuclear lamina-genome interactions are highly conserved and associated with A/T-rich sequence. Genome Res. 23, 270-280. doi: 10.1101/ gr.141028.112

Mohandas, T., Sparkes, R. S., and Shapiro, L. J. (1981). Reactivation of an inactive human $\mathrm{X}$ chromosome: evidence for $\mathrm{X}$ inactivation by DNA methylation. Science 211, 393-396.

Mudbhary, R., Hoshida, Y., Chernyavskaya, Y., Jacob, V., Villanueva, A., Fiel, M. I., et al. (2014). UHRF1 overexpression drives DNA hypomethylation and hepatocellular carcinoma. Cancer Cell 25, 196-209. doi: 10.1016/j.ccr.2014. 01.003

Ong, C. T., and Corces, V. G. (2014). CTCF: an architectural protein bridging genome topology and function. Nat. Rev. Genet. 15, 234-246. doi: 10.1038/ nrg3663

Peric-Hupkes, D., Meuleman, W., Pagie, L., Bruggeman, S. W., Solovei, I., Brugman, W., et al. (2010). Molecular maps of the reorganization of genomenuclear lamina interactions during differentiation. Mol. Cell. 38, 603-613. doi: 10.1016/j.molcel.2010.03.016

Pujadas, E., and Feinberg, A. P. (2012). Regulated noise in the epigenetic landscape of development and disease. Cell 148, 1123-1131. doi: 10.1016/j.cell.2012.02.045

Reddy, K. L., Zullo, J. M., Bertolino, E., and Singh, H. (2008). Transcriptional repression mediated by repositioning of genes to the nuclear lamina. Nature 452, 243-247. doi: 10.1038/nature06727

Reik, W., Collick, A., Norris, M. L., Barton, S. C., and Surani, M. A. (1987). Genomic imprinting determines methylation of parental alleles in transgenic mice. Nature 328, 248-251. doi: 10.1038/328248a0

Roadmap Epigenomics, C., Kundaje, A., Meuleman, W., Ernst, J., Bilenky, M., Yen, A., et al. (2015). Integrative analysis of 111 reference human epigenomes. Nature 518, 317-330. doi: 10.1038/nature14248

Robson, M. I., de Las Heras, J. I., Czapiewski, R., Le Thanh, P., Booth, D. G., Kelly, D. A., et al. (2016). Tissue-specific gene repositioning by muscle nuclear membrane proteins enhances repression of critical developmental genes during myogenesis. Mol. Cell. 62, 834-847. doi: 10.1016/j.molcel.2016.04.035

Rodriguez, J., Frigola, J., Vendrell, E., Risques, R. A., Fraga, M. F., Morales, C., et al. (2006). Chromosomal instability correlates with genome-wide DNA demethylation in human primary colorectal cancers. Cancer Res. 66, 8462-9468. doi: 10.1158/0008-5472.CAN-06-0293

Rose, N. R., and Klose, R. J. (2014). Understanding the relationship between DNA methylation and histone lysine methylation. Biochim. Biophys. Acta 1839, 1362-1372. doi: 10.1016/j.bbagrm.2014.02.007

Sapienza, C., Peterson, A. C., Rossant, J., and Balling, R. (1987). Degree of methylation of transgenes is dependent on gamete of origin. Nature 328, 251-254. doi: 10.1038/328251a0

Schwartz, D., and Dennis, E. (1986). Transposase activity of the Ac controlling element in maize is regulated by its degree of methylation. Mol. Gen. Genet. 205, 476-482. doi: 10.1007/bf00338085

Shevelyov, Y. Y., Lavrov, S. A., Mikhaylova, L. M., Nurminsky, I. D., Kulathinal, R. J., Egorova, K. S., et al. (2009). The B-type lamin is required for somatic repression of testis-specific gene clusters. Proc. Natl. Acad. Sci. U.S.A. 106, 3282-3287. doi: 10.1073/pnas.0811933106 
Swain, J. L., Stewart, T. A., and Leder, P. (1987). Parental legacy determines methylation and expression of an autosomal transgene: a molecular mechanism for parental imprinting. Cell 50, 719-727.

Timp, W., Bravo, H. C., McDonald, O. G., Goggins, M., Umbricht, C., Zeiger, M., et al. (2014). Large hypomethylated blocks as a universal defining epigenetic alteration in human solid tumors. Genome Med 6:61. doi: 10.1186/s13073-0140061-y

Timp, W., and Feinberg, A. P. (2013). Cancer as a dysregulated epigenome allowing cellular growth advantage at the expense of the host. Nat. Rev. Cancer 13, 497-510. doi: 10.1038/nrc3486

Trinh, B. N., Long, T. I., Nickel, A. E., Shibata, D., and Laird, P. W. (2002). DNA methyltransferase deficiency modifies cancer susceptibility in mice lacking DNA mismatch repair. Mol. Cell. Biol. 22, 2906-2917.

Tubio, J. M., Li, Y., Ju, Y. S., Martincorena, I., Cooke, S. L., Tojo, M., et al. (2014). Mobile DNA in cancer. Extensive transduction of nonrepetitive DNA mediated by L1 retrotransposition in cancer genomes. Science 345:1251343. doi: 10.1126/science.1251343

Varley, K. E., Gertz, J., Bowling, K. M., Parker, S. L., Reddy, T. E., Pauli-Behn, F., et al. (2013). Dynamic DNA methylation across diverse human cell lines and tissues. Genome Res. 23, 555-567. doi: 10.1101/gr.147942.112

Wen, B., Wu, H., Loh, Y. H., Briem, E., Daley, G. Q., and Feinberg, A. P. (2012). Euchromatin islands in large heterochromatin domains are enriched for CTCF binding and differentially DNA-methylated regions. BMC Genomics 13:566. doi: 10.1186/1471-2164-13-566

Wen, B., Wu, H., Shinkai, Y., Irizarry, R. A., and Feinberg, A. P. (2009). Large histone $\mathrm{H} 3$ lysine 9 dimethylated chromatin blocks distinguish differentiated from embryonic stem cells. Nat. Genet. 41, 246-250. doi: 10.1038/ ng. 297

Zhang, C., Hoshida, Y., and Sadler, K. C. (2016). Comparative epigenomic profiling of the DNA methylome in mouse and zebrafish uncovers high interspecies divergence. Front. Genet. 7:110. doi: 10.3389/fgene.2016. 00110

Ziller, M. J., Gu, H., Muller, F., Donaghey, J., Tsai, L. T., Kohlbacher, O., et al. (2013). Charting a dynamic DNA methylation landscape of the human genome. Nature 500, 477-481. doi: 10.1038/nature 12433

Zink, D., Fischer, A. H., and Nickerson, J. A. (2004). Nuclear structure in cancer cells. Nat. Rev. Cancer 4, 677-687. doi: 10.1038/nrc1430

Zullo, J. M., Demarco, I. A., Pique-Regi, R., Gaffney, D. J., Epstein, C. B., Spooner, C. J., et al. (2012). DNA sequence-dependent compartmentalization and silencing of chromatin at the nuclear lamina. Cell 149, 1474-1487. doi: 10.1016/j.cell.2012.04.035

Conflict of Interest Statement: The authors declare that the research was conducted in the absence of any commercial or financial relationships that could be construed as a potential conflict of interest.

Copyright (C) 2017 Madakashira and Sadler. This is an open-access article distributed under the terms of the Creative Commons Attribution License (CC BY). The use, distribution or reproduction in other forums is permitted, provided the original author(s) or licensor are credited and that the original publication in this journal is cited, in accordance with accepted academic practice. No use, distribution or reproduction is permitted which does not comply with these terms. 